\title{
Supporting information for Photolithographic Fabrication of Semi 3-D Microstructures Composed of Flexible Hydrogel Sheet for in Vivo-like Cell Culture System
}

\begin{abstract}
Atsushi Tsuyukubo1,2, Kimio Sumaru1*, and Toshiyuki Kanamori1,2
1Biotechnology Research Institute for Drug Discovery (BRD), National Institute of Advanced Industrial

Science and Technology (AIST), Tsukuba Central fifth, 1-1-1 Higashi, Tsukuba, Ibaraki 305-8565, Japan

2School of Integrative and Global Majors, University of Tsukuba, 1-1-1 Tennodai, Tsukuba, Ibaraki 305-

8577, Japan
\end{abstract}

*E-mail: k.sumaru@ aist.go.jp 


\section{Materials}

Hydroxypropyl cellulose (HPC, M.W. 100,000, \#19188-4, Sigma-Aldrich Co.) was used as a watersoluble hydroxyl-rich polymer composing hydrogel sheet. 1,3,4,6-tetrakis (methoxymethyl) glycoluril (TMMGU, \#T2058, Tokyo Chemical Industry Co., Ltd.) was used as an acid-catalyzed cross-linker. NAI106 (Midori Kagaku Co., Ltd.) was used as a UV responsive photoacid generator (PAG). Poly (acrylic acid) (PAAc, M.W. 250,000, \#169-18591, FUJIFILM Wako Pure Chemical Corp.) was used as a polymer composing sacrificial thin layer. Polystyrene petri dish (\#3000-035, AGC TECHNO GLASS Co., Ltd.) with $35 \mathrm{~mm}$ diameter was used as a basal substrate. Rhodamine B isothiocyanate (RITC, \#R1755, SigmaAldrich Co.) was used to prepare a fluorescently-functionalized HPC (fHPC). Fluorescent diacetate (FLDA, \#F0240, Tokyo Chemical Industry Co., Ltd.) was used to observe cell fluorescence. Poly (styrene-co-maleic anhydride) (PSMA, 75:25, M.W. 1,900, \#03500, PolySciences, Inc.) was used as a polymer providing HPC hydrogel sheet with cell adhesive property. Collagen (Cellmatrix Type I-C, Nitta Gelatin Inc.) was used to coat on the microstructure and improve HepG2 cell adhesiveness. Chitosan (Chitosan 10B, \#KB-004, Funakoshi Co., Ltd.) was used as a polymer inhibiting cell adhesion on the substrate surface inside the microstructure. HeLa cells, HepG2 cells and Madin-Darby canine kidney cells were provided by the RIKEN Bioresource center (Tsukuba, Ibaraki, Japan).

\section{Apparatus}

A spin-coater (ASS-301, Able Co., Ltd.) was used for coating polymer solutions. Micro-patterned UV light irradiation (wavelength: $365 \mathrm{~nm}$, intensity: $1 \mathrm{~W} / \mathrm{cm}_{2}$ ) was carried out by using a PC-controlled microprojection system (DESM-01, Engineering System Co.) installed in an inverted research microscope (IX70, Olympus Co.) through a 4X objective lens (PlanApo 4X, Olympus Co.)1,2. Bright field images were taken with a cooled CCD camera system (VB-7000, Keyence Co.) installed on the same microscope. 3dimentional and fluorescent observation of the microstructures was carried out by using a confocal laser scanning microscope (Fluoview 300, Olympus Co.) installed in inverted research microscope (IX71, Olympus Co.). A UV-Vis spectrometer (V-560, Jasco Co.) was used for measuring absorbance of cHPC. A UV light stand (UV365STND, OptoCode Corp.) was used for preparing cHPC.

\section{Cell incubation}

All cells used in this study were maintained and repeatedly subcultured in Minimum Essential Medium Eagle (E-MEM, \#M4655, Sigma-Aldrich Co.) containing 10\% FBS, penicillin, streptomycin, Sodium Pyruvate solution (\#190-14881, FUJIFILM Wako Pure Chemical Corp.) and MEM Non-essential Amino Acids solution (\#139-15651, FUJIFILM Wako Pure Chemical Corp.) at $37^{\circ} \mathrm{C}$ in the incubator (humidified $95 \%$ air, $5 \% \mathrm{CO}_{2}$ atmosphere). 


\section{Synthesis of fHPC}

HPC and RITC were dissolved in acetone by using supersonic waves. fHPC was synthesized by heating to dry the acetone solution containing $0.98 \mathrm{wt} \% \mathrm{HPC}$ and $0.082 \mathrm{wt} \% \mathrm{RITC}$ at $80{ }^{\circ} \mathrm{C}$ for 30 minutes. fHPC was dissolved in methanol and the solution was used to prepare pre-gel solution.

\section{Preparation of cHPC layer}

A TFE solution containing $0.80 \mathrm{wt} \% \mathrm{HPC}, 0.019 \mathrm{wt} \%$ TMMGU and $0.018 \mathrm{wt} \%$ NAI-106 was spincoated on the surface of the PS dish at 2,000 rpm. The coated layer was irradiated with UV light (wavelength: $365 \mathrm{~nm}$, Intensity: $8 \mathrm{~mW} / \mathrm{cm}_{2}$ ) by using the UV light stand for 30 minutes, and was baked at $85^{\circ} \mathrm{C}$ for one and a half hours. After flushing with water, cHPC layer was obtained.

\section{Fluorescence observation of cultured cells}

An ethyl acetate solution containing $0.31 \mathrm{wt} \%$ FLDA was prepared. An ethanol solution containing $0.0098 \mathrm{wt} \%$ FLDA was prepared by diluting the ethyl acetate solution with ethanol. $5 \mu \mathrm{L}$ of the ethanol solution was added to $2 \mathrm{~mL}$ HeLa cells culture medium, and laid it at the incubator for 30 minutes. It was suggested that FLDA molecules taken into these cells were hydrolyzed by their esterase and fluorescein was generated in them 3 .

\section{Statistical analysis}

Data of absorbance were presented by mean \pm standard deviation $(n=4)$. Statistical comparison was performed using Student's t-test. Differences of $\mathrm{p}<0.05$ were considered to be statistically significant. All error bars were presented as standard deviation. (Figure 4)

\section{Supporting movie}

Animations to illustrate each pocket-like hydrogel microstructure can be seen in the movies mt9b00656_si_003.avi and mt9b00656_si_004.avi. Moreover, an animation to illustrate semi 3-D culture system composed of HeLa cells also can be seen in the movie mt9b00656_si_005.avi.

\section{Difference between the microstructures corresponding to each condition (irradiation time to cross-link PAAc and concentration of cross-linker in pre-gel solution)}

Figure S1 shows that it was easier for cHPC sheet to lift off of the substrate as the irradiation time to cross-link PAAc was larger. But, cHPC sheet lifted off not only in the irradiated area but also in other area if dose was too large. It is because slight light was irradiated outside of irradiation pattern due to the characteristic of the apparatus. And when irradiation pattern was mesh design, it was hard for cHPC sheet to lift off compared with flat design. 
Considering difference between (a) and (b), it was observed that it was more difficult for cHPC sheet to lift off of the substrate as TMMGU concentration in pre-gel solution was higher. Moreover, it was also observed that swelling ratio of HPC hydrogel sheet was lower suggesting that this was caused by increasing cross-linking density due to high TMMGU concentration. The best irradiation time in (a) was 240 s while that in (b) was $60 \mathrm{~s}$.

(a) High TMMGU concentration
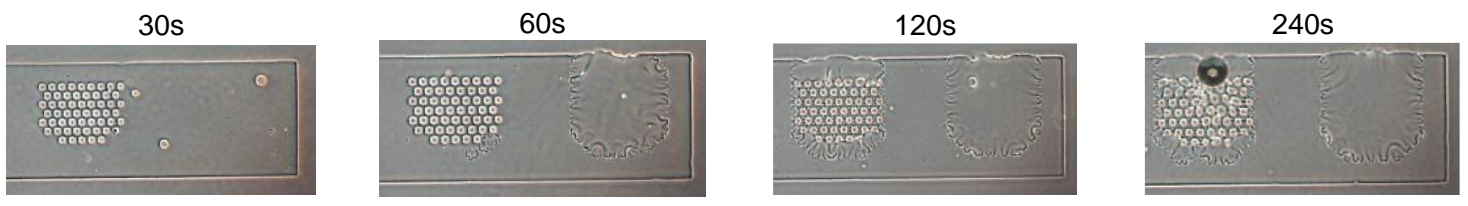

(b) Low TMMGU concentration
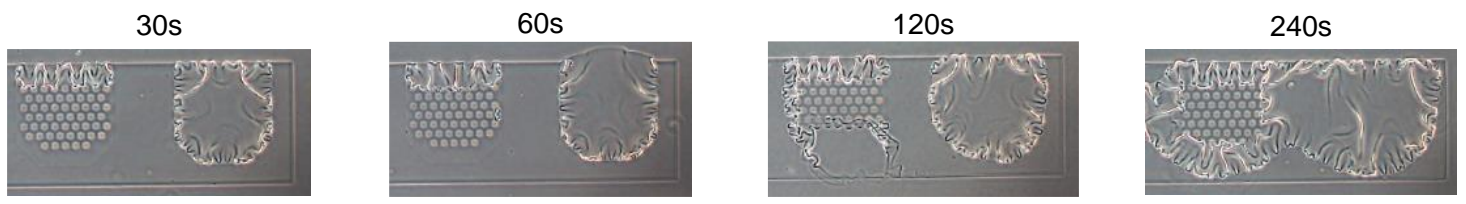

Figure S1. HPC hydrogel microstructures after soaking in $1 \mathrm{M} \mathrm{NaOH}$ aqueous solution in a day corresponding to each irradiation time to cross-link PAAc. (a) High TMMGU concentration, (b) Low TMMGU concentration

\section{Detailed conditions for fabrication of the microstructures}

Detailed conditions (concentration and amount of coated solution, rotation speed for spin-coating and irradiation time) corresponding to the microstructure fabricated are summarized in Table S1.

\section{References}

(1) Sumaru, K.; Edahiro, J.; Ooshima, Y.; Kanamori, T.; Shinbo, T. Manipulation of living cells by using PC-controlled micro-pattern projection system. Biosens. Bioelectron. 2007, 22, 2356-2359.

(2) Sumaru, K.; Kanamori, T. Cell Patterning by Micro-Pattern Projection of UV Light Through Photoinduced Enhancement of Cell Adhesion (PIECA). Methods Cell Biol. 2014, 120, 185-197.

(3) Rotman, B.; Papermaster, B. W. Membrane properties of living mammalian cells as studied by enzymatic hydrolysis of fluorogenic esters. Proc. Natl. Acad. Sci. USA 1966, 55, 134-141. 
Table S1. Detailed conditions for fabrication of the microstructures.

\begin{tabular}{|c|c|c|c|c|c|c|c|c|c|c|c|c|}
\hline & \multicolumn{5}{|c|}{ cPAAc layer } & \multicolumn{7}{|c|}{ cHPC layer } \\
\hline & \multicolumn{2}{|c|}{$\begin{array}{c}\text { Concentration of } \\
\text { solutes }(\mathrm{wt} \%)\end{array}$} & \multirow{2}{*}{$\begin{array}{c}\text { Amount of } \\
\text { solution } \\
(\mu \mathrm{L})\end{array}$} & \multirow{2}{*}{$\begin{array}{l}\text { Rotation } \\
\text { speed } \\
(\mathrm{rpm})\end{array}$} & \multirow{2}{*}{$\begin{array}{l}\text { Irradiation } \\
\text { time (s) }\end{array}$} & \multicolumn{4}{|c|}{ Concentration of solutes (wt\%) } & \multirow{2}{*}{$\begin{array}{c}\text { Amount of } \\
\text { solution } \\
(\mu \mathrm{L})\end{array}$} & \multirow{2}{*}{$\begin{array}{c}\text { Rotation } \\
\text { speed } \\
(\mathrm{rpm})\end{array}$} & \multirow{2}{*}{$\begin{array}{l}\text { Irradiation } \\
\text { time }(\mathrm{s})\end{array}$} \\
\hline & PAAc & NAI-106 & & & & $\mathrm{HPC}$ & fHPC & TMMGU & $\begin{array}{c}\text { NAI- } \\
106\end{array}$ & & & \\
\hline Pocket $1 * 1$ & 0.39 & 0.0049 & 15 & 1,500 & 240 & 2.8 & 0.044 & 0.14 & 0.092 & 60 & 2,000 & 180 \\
\hline Pocket $2 * 3$ & 0.38 & 0.0044 & 15 & 1,500 & 240 & 3.2 & 0.051 & 0.070 & 0.091 & 100 & 2,000 & 150 \\
\hline Pocket $3 * 4$ & 0.38 & 0.0035 & 10 & 1,500 & 60 & 3.1 & 0.048 & 0.076 & 0.093 & 70 & 2,000 & 180 \\
\hline Pocket $4 * 5$ & 0.38 & 0.0034 & 15 & 1,500 & 240 & 3.3 & 0 & 0.073 & 0.094 & 80 & 2,000 & 120 \\
\hline Pocket $5 * 6$ & 0.39 & 0.0049 & 15 & 1,500 & $\begin{array}{c}30,60 \\
120,240\end{array}$ & 2.8 & 0.044 & 0.14 & 0.092 & 60 & 2,000 & 180 \\
\hline Pocket6*7 & 0.38 & 0.0035 & 10 & 1,500 & $\begin{array}{c}30,60, \\
120,240\end{array}$ & 3.1 & 0.048 & 0.076 & 0.093 & 60 & 2,000 & 180 \\
\hline
\end{tabular}

*1. Pocket-like microstructure corresponding to Figure 2, Figure 3(a)(1), (2) and Figure 3(b)

*2. String-like microstructure corresponding to Figure 3(a)(3).

*3. Semi 3-D culture system composed of HeLa cells corresponding to Figure 5(a), (d). $50 \mu \mathrm{L}$ of HFIP solution containing 0.096wt\% PSMA was over-coated on the patterned cHPC sheet at 1,500 rpm. The obtained microstructure was soaked in $\mathrm{HCl}$ aqueous solution containing $1.0 \mathrm{wt} \%$ chitosan. After flushing with water, HeLa cells were seeded and incubated.

*4. Semi 3-D culture system composed of MDCK cells corresponding to Figure 5(b). $30 \mu \mathrm{L}$ of a HFIP solution containing 0.094 wt\% PSMA was over-coated on the cHPC sheet at 1,500 rpm. After flushing with water, MDCK cells were seeded and incubated.

*5. Semi 3-D culture system composed of HepG2 cells corresponding to Figure 5(c). $50 \mu \mathrm{L}$ of a HFIP solution containing 0.095 wt $\%$ PSMA was over-coated on the cHPC sheet at 1,500 rpm. The obtained pocket microstructure was soaked in $1 \mathrm{mM} \mathrm{HCl}$ aqueous solution containing 1 vol\% collagen for $30 \mathrm{minutes}$ in room temperature. After flushing with D-PBS, HepG2 cells were seeded and incubated.

*6. Pocket-like microstructure corresponding to Figure S1(a) High TMMGU concentration.

*7. Pocket-like microstructure corresponding to Figure S1(b) Low TMMGU concentration. 